\title{
A Novel Way of Storage and Recycling of the Context Information Data
}

\author{
Younghwan Oh \\ Professor, Department of Information Communication, Korea Nazarene University \\ yhoh@kornu.ac.kr
}

\begin{abstract}
The things to realize using the network system of ubiquitous sensors are situation perception and personalization services. To enhance performance of the situation perception, construct wireless networks with multi-sensors. In other to enhance the performance of the context awareness, temporary storage and utilization for the sensing data were proposed. The criteria to judge whether to use or not the sensing data reported from the sensor in the next time zones was presented by saving it temporarily, the data proved to be used in the next time zones should be recycled. On using by calling the Basic Probability Assignment saved, weights corresponding to the increment of the previous and present time zones should be added. Since then, the belief and uncertainty were calculated by using each focal element's $B P A$ and through this, advanced information can be deduced rather than obtaining context information.
\end{abstract}

Keywords: Context Awareness, Context Inference, Data fusion

\section{Introduction}

The things to realize using the network system of ubiquitous sensors are situation perception and personalization services. To enhance performance of the situation perception, construct wireless networks with multi-sensors. Data which a lot of sensors collect in the sensor networks can make the situation perception realized through proper analyses. In here, the situation can be said information identified by characterizing really existing states.

For the situation information, first, user situation; second physical environmental situation; third, computer system situation; forth, computer interaction history; and fifth, other nonclassified situations are divided [1,2]. For the situation information to express classified situations like these, the kinds are so many and the amounts of information to be collected and dealt with are very tremendous. The system to perceive these situations needs consecutive monitoring and complex calculation competency. The top priority is situation information sensing in order to perceive these situations. Basic situation information is collected from users or sensors attached to user surroundings. The situation information sensing is used for data of situation deduction later after going through a course to save the collected situation information into the inside[3].

Upper rank information is induced by blending diverse sensing data, and this is called situation information deduction. Though various deduction ways of situation information have been studied, the most used thing is the way using ontology. By using the ontology, situation knowledge is shared, reuse support is possible, and the deduction of situation information is executed. The deduction on the basis of the ontology is to deduce the situation information by analogizing knowledge and facts obtained from the ontology [4]. 
The basis here is to model the situation information. Although there are purposes that situation information can be reused or exchanged, since the situation information modeling can be a foundation which the deduction of the situation information based on the ontology is possible, it is necessary that high reliable modeling can be made through enough previous information. But, it will be very massive work to acquire information previously and model these about too many situations [5].

Therefore, the situation information modeling cannot avoid narrowing its range into local space and time related with situations for goals to be set rather than comprehensive ranges about all situations of the natural world and real world. It is difficult to say modeling all situations within the ranges although modeling is done by narrowing the ranges. Among a lot of situations happening really, there are things that are out of the ranges of modeling [6, 7].

This study is set in a condition to cope with a situation that gets out of the previous modeling ranges. When deducing the situation information in the course for situation perception using wireless sensor networks. As subsidiary methods to deal with a situation to go out of the ranges of previous information modeled like the ontology and to supplement weak points of the deduction of previous situation information, it is necessary to consider a plan even possible for the deduction of the situation information by evaluating changes and features of values targeting sensing signals.

Like those, not referring to data models grounded on previous information, when situation information is deducted by evaluating meanings and worth, there are points to be careful. So, it is to think about supplementary points after looking into deduction courses of the situation information on the basis of primary signals.

There is a case of relying on sensing data itself beyond the scope of pre-modeling in the context awareness using multiple sensors. There are a case of serious or urgent event and a case that the situation is difficult to obtain information in advance. In addition, when getting the data reported from the sensor, the thing to update the information related to the real situation occurs. It's updated over time later on but, there is a case of utilizing urgently sensing data. Then, there is a need to have interest in the process of reasoning the situation. When the sensor detects and reports the information on the surrounding situation or the object which the sensor is attached on, there is a case that the real situation that people want to recognize through the sensor is made on single shot, but there are a lot of cases that it's persisted or changed for a certain period of time. Therefore, the sensor is to detect repeatedly and report consistently as time passes. In such an environment, situation reasoning can be performed in each time with fusion processing of the sensing data obtained in that time, but there is a case of recycling previous sensing data in the following time zones. In other words, there is a case of processing the data including the values measured in the previous time zones as well as processing the data for situation reasoning with only current input values. Like this, for a case of utilizing the values measured and reported in the previous time zones in the situation not to be modeled in advance, this study suggests plans of temporary storage and using for sensing data. To this end, it should be judged whether to utilize the sensing data reported from the sensor in the following time zones by saving it temporarily. For the data proved to be the sensing data that people should use in the following time zones, its values should be used for fusion processing. The details about whether to save the data detected and reported in the previous time zones at this time in any form should be provided. In this study, on constant use target for the sensing data in each time zones was selected, it was to deduce advanced information rather than obtain situation information using multiple sensors by proposing ways to save to this end.

This paper is described as follows: In Chapter 2, technology-related research is described, in Chapter 3, sensing data storage and use plans for continuous utilization suggested in this 
study are described. In Chapter 4, experiments and evaluation are described, in Chapter 5, it's concluded.

\section{Related Research}

Meanwhile, diverse studies have been realized for the situation perception, if the studies of the situation perception are arranged in several aspects, they are like the next.

\section{Situation Information Modeling}

Strang [8] said that six demands should be fulfilled for models of the situation perception; and they are distributed composition, partial validation, richness and quality of information, incompleteness and ambiguity, level of formality, and application to existing environments. And, as models of the situation information, there are key-value models, markup scheme models, graphical models, logic based models, object oriented models, ontology based models, and so on.

Strang analyzed how diverse situation information models could reflect these six demands. As a result, the models of situation perception based on the ontology in all aspects were presented to be excellent. Grounded on this result, lots of researchers are developing situation perception modeling on the basis of the ontology, standardization activities on that are realized together [9].

\section{Situation Information Perception}

Hoolet[10] was manufactured on the basis of vampire prover for parsing of OWL and process and deduction of situation information, and it is a deduction engine easy for expansion.

F-OWL[11] is a private deduction engine for OWL Lite ontology and manufactured based on Flora-2. The Flora-2 is an object-oriented knowledge-based language and application development platform that a united language of F-login, HiLog, and transaction logic are transformed into a XSB deduction engine.

Jena2[12] is a Java framework written for an application for a semantic web and its physical schema structure is simple in the automatic generation of a saving model. Owing to this, this is used in lots of OWL-related system development.

\section{Middleware and Service Model for Context-awareness}

GAIA[13] is a service structure of the situation perception developed in University of Illinois. It can make the situation information which is capable of diverse applications obtained and deducted. The GAIA system can make services exposed into the outside for the purpose of querying and using existing resources, and simultaneously accessing and using situation information. This is user-oriented and possible for resource perception, and it can use several devices and provides a framework available to use the situation information effectively.

SOCAM[14] which was developed at Singapore national university is a middleware and was proposed to ease situation service and the system development. In the middleware, OWL for the situation information modeling was used. After acquiring the situation information from dispersed situation information providers, by processing it in proper types, a central server called context interpreter to provide information to the situation perception service is used. It is composed of providers and translators of the situation information, database of the situation information, services of the situation information, and location-service components. 
As seeing the diverse situation perception researches like the upper contents, it is difficult to search that coping plans for situations that previous modeling ranges are out of are prepared specifically. Since the situation that gets out of the previous modeling ranges sometimes incur serious results or situations to be taken notice of, the coping methods for the problems in situation perception studies should be devised.

Context Toolkit [1][2] suggested by Georgia Tech is widget-based dispersion system and attempted data concealment from superior layer after saving data acquired by sensors as consistent widget patterns. Detecting inferior level context information was differentiated from superior level service conceptually. Middle media managing contexts were used to solve problems in the possibility of context reuse and the rapidity among sensors' application services.

Hydrogen [15] included three layers of adaptor, management, and application. The layer of adaptor searches raw data from sensors and uses multiple application sensors. The management layer transmits context information using raw data and supports a search. The context information server provides services with saved information. The application layer provides the service on demand for particular context information. The object-oriented strengths of capsulizing and reuse are applied to solve problems in dynamic characteristics of circumstances. Hydrogen is also a limited framework to interpret high level context information.

\section{Sensing Data Storage and Use Plans for Continuous Utilization}

\section{Multi-sensor Network System and Data Fusion}

The research on obtaining the information about the object or sensor that the sensor is attached on by using the sensor and the environment of the object has actively been conducted. The information collected with a heterogeneous multi-sensor contributes to situation information compared to the information collected with a single sensor. However, this information should be fused to take full advantage of the information from the heterogeneous multi-sensor. The procedures of the data fusion process are different from those of the data merge and its goal to purse is different. The data merge's goal is to save resources of the network system and the data merge is mainly done with de-duplication. The data fusion is to use stochastic methods, learning-based fusion or Fuzzy theory by extracting high-level information with the fragmentary data fusion.

\section{The Data Fusion and Time for Context Awareness}

It's the context information that the sensor network system tries to acquire through a multisensor's detecting activities and reporting. Time is not a factor considered in case of improving geography information and the quality of medical imaging information through the multi-sensor data fusion. However, the passage of time should be taken into account such as shifting unmanned air vehicles, robots, desperate danger occurred in the real life. The situation can be changed as time goes by. It can be a very different situation and this phase of the new situation can be appeared as time passes. Thus, it's important to judge whether to consider time in context awareness or not. The measure to consider time in context awareness is to use Kalman equations, which is mainly used as a purpose for recognizing the location of moving objects. Alternately, it's to reason situation by fusing data sensed and reported in each time by appointing a certain period of time. 


\section{Sensing Data Storage and use for Continuous Utilization}

In this study, when the multi-sensor data fusion for situation reasoning is conducted by using sensing data at regular intervals like this, in case of using the sensing data obtained from the previous time zones not to take advantage of only data got at that time, it suggests the plan on how to save the sensing data obtained in the previous time zones and the plan to utilize the data fusion executed in the next time zones.

\section{Storage Plans}

The sensing data that each sensor detects and reports is the result of a data filtering policy which each sensor reports as an event. This data holds units as measurements and physical properties. In this paper, if the data is reflected on the data fusion conducted in the next time zones, it's proposed to save the data in BPA form. When the multi-sensor data fusion is carried out for context awareness in each time zones, BPA in each focal element based on the sensing data is calculated and focal element's belief and uncertainty based on this is done and then, situation is deduced. Therefore, it's easy for the data fusion in the next time zones and reduce cost to save the sensing data utilized in the previous time zones in BPA form in order to use previous data in the following time zones. It should be noted here, to determine whether to save any data or not. Basically it's a case of reducing event incidence significantly. However, in this study, it suggests to do by changes in the pattern of events. It's each Focal element's belief value to note in changes in the pattern. the data which the sensor involved in focal element that the belief value increase linearly detected is sure to be saved. The belief value is calculated from BPA of the focal element, the meaning of the belief value is appropriate to certain probability that the event occurs. In other words, it's the value to represent the certainty of the incident as probable values. Therefore, if the belief value increases compared to the previous time zones, the sensor data involved in the facal element is an object of observation and should be utilized in the next time zones by saving the value. The following figure shows the context awareness model of the multi-sensor system.

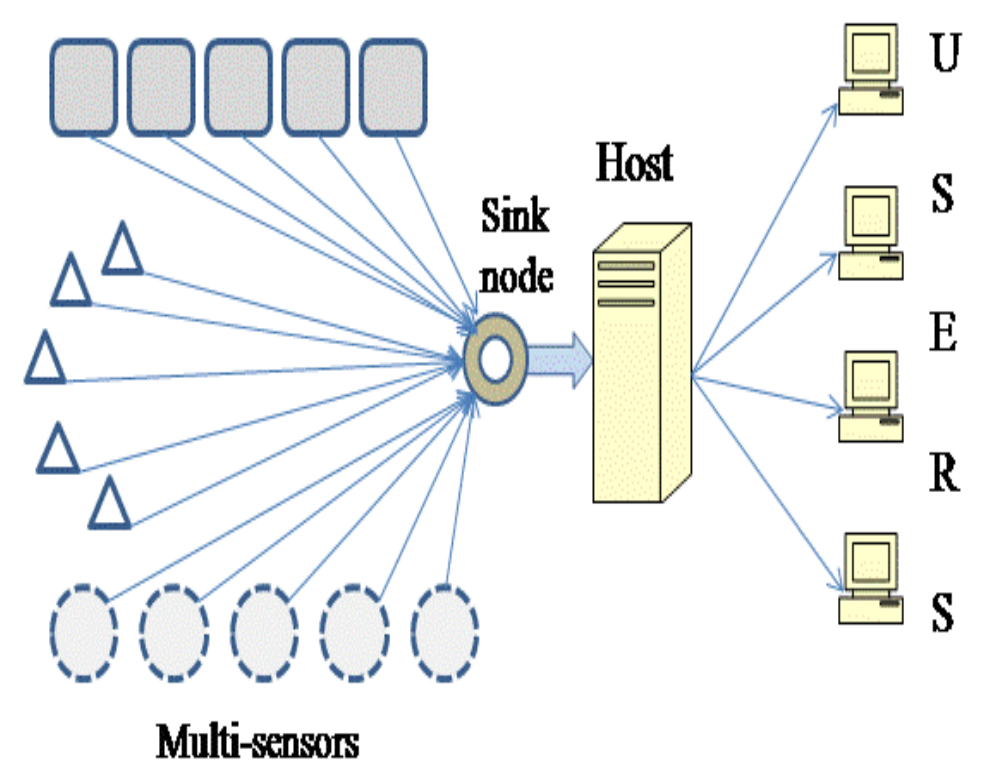

Figure 1. Context Awareness of the Multi-sensor System 
The following figure illustrates sensing data storage and use plans

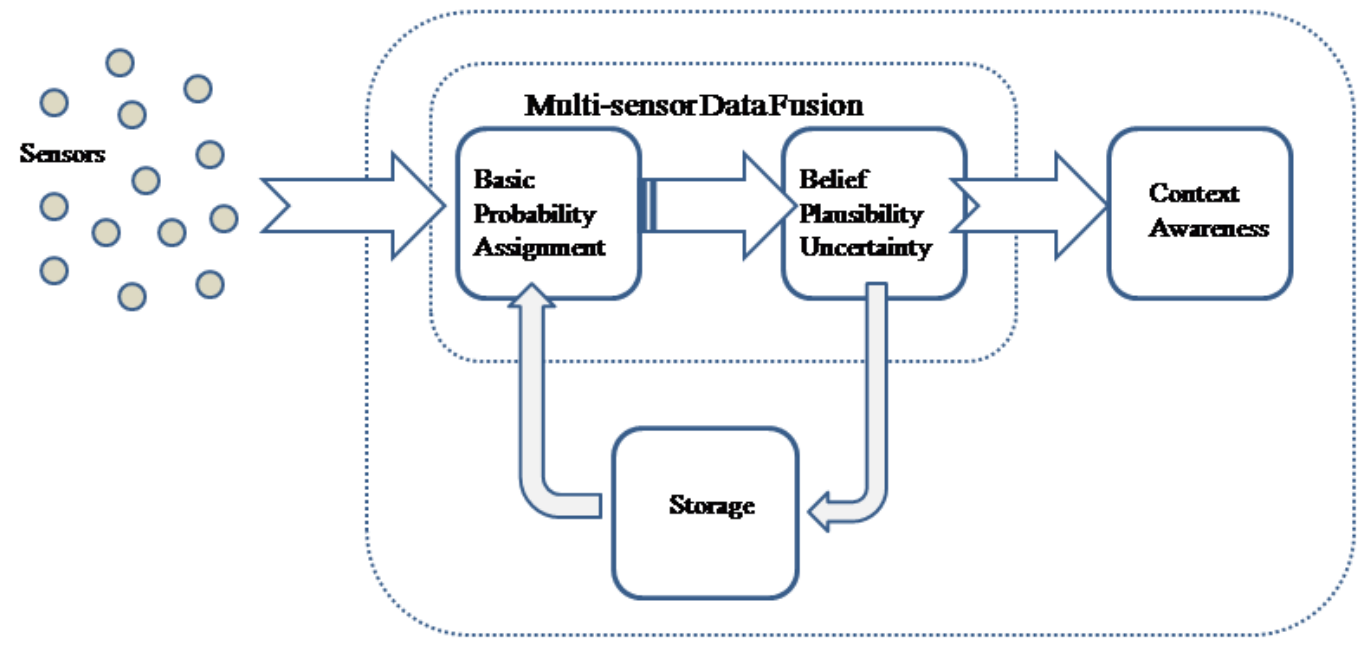

Figure 2. Sensing Data Storage and use for Continuous Utilization

Next, it's about the plan how to recycle the sensing data saved temporarily by these conditions. In this paper, BPA of the sensing data utilized again in the next time zones and the current average of BPA are used to obtain, weights as much as the amount increased by comparing two values are added. Through this, the proportion of the focal element should be reflected on context awareness.

\section{Experiments and Evaluation}

Author names and Experiments contents: In the situation that events of the increase in temperature occurs, it's to detect and save the belief value of the focal element related to temperature sensors increases and then to do fusion processing with the BPA value in the next time zones, to conduct context reasoning.

The following table is the value of the BPA value of the focal element in each time

Table 1. Focal Element's BPA

\begin{tabular}{cccccc}
\hline $\begin{array}{c}\text { Focal } \\
\text { element }\end{array}$ & $\mathrm{m}\left(\mathrm{T}^{\prime} 0\right)$ & $\mathrm{m}\left(\mathrm{T}^{\prime} 1\right)$ & $\mathrm{m}\left(\mathrm{T}^{\prime} 2\right)$ & $\mathrm{m}\left(\mathrm{T}^{\prime} 3\right)$ & $\mathrm{m}\left(\mathrm{T}^{\prime} 4\right)$ \\
\hline$\Omega$ & 0.28 & 0.30 & 0.10 & 0.00 & 0.00 \\
$\mathrm{~h} 1 \cup \mathrm{h} 2$ & 0.19 & 0.05 & 0.10 & 0.00 & 0.00 \\
$\mathrm{~h} 1 \cup \mathrm{h} 3$ & 0.01 & 0.15 & 0.05 & 0.10 & 0.09 \\
$\mathrm{~h} 2 \cup \mathrm{h} 3$ & 0.15 & 0.20 & 0.16 & 0.19 & 0.16 \\
$\mathrm{~h} 1$ & 0.07 & 0.03 & 0.10 & 0.11 & 0.15 \\
$\mathrm{~h} 2$ & 0.12 & 0.00 & 0.13 & 0.15 & 0.13 \\
h3 & 0.17 & 0.27 & 0.36 & 0.44 & 0.45 \\
\hline
\end{tabular}


The following table is to summarize the belief and uncertainty of the Focal element calculated after fusion in the next time zones by calling the temporary storage value[16, 17].

Table 2. Fusion Results in each Interval

\begin{tabular}{cccccc}
\hline $\begin{array}{c}\text { Focal } \\
\text { element }\end{array}$ & bel(T’0) & $\operatorname{bel}\left(\mathrm{T}^{\prime} 1\right)$ & $\operatorname{bel}\left(\mathrm{T}^{\prime} 2\right)$ & $\operatorname{bel}\left(\mathrm{T}^{\prime} 3\right)$ & $\operatorname{bel}\left(\mathrm{T}^{\prime} 4\right)$ \\
\hline$\Omega$ & 1.00 & 1.00 & 1.00 & 1.00 & 1.00 \\
$\mathrm{~h} 1 \cup \mathrm{h} 2$ & 0.39 & 0.08 & 0.33 & 0.27 & 0.29 \\
$\mathrm{~h} 1 \cup \mathrm{h} 3$ & 0.25 & 0.45 & 0.51 & 0.66 & 0.70 \\
$\mathrm{~h} 2 \cup \mathrm{h} 3$ & 0.44 & 0.47 & 0.65 & 0.78 & 0.75 \\
$\mathrm{~h} 1$ & 0.07 & 0.03 & 0.10 & 0.11 & 0.15 \\
$\mathrm{~h} 2$ & 0.12 & 0.00 & 0.13 & 0.15 & 0.13 \\
h3 & 0.17 & 0.27 & 0.36 & 0.44 & 0.45 \\
\hline
\end{tabular}

The following graph illustrates the above table.

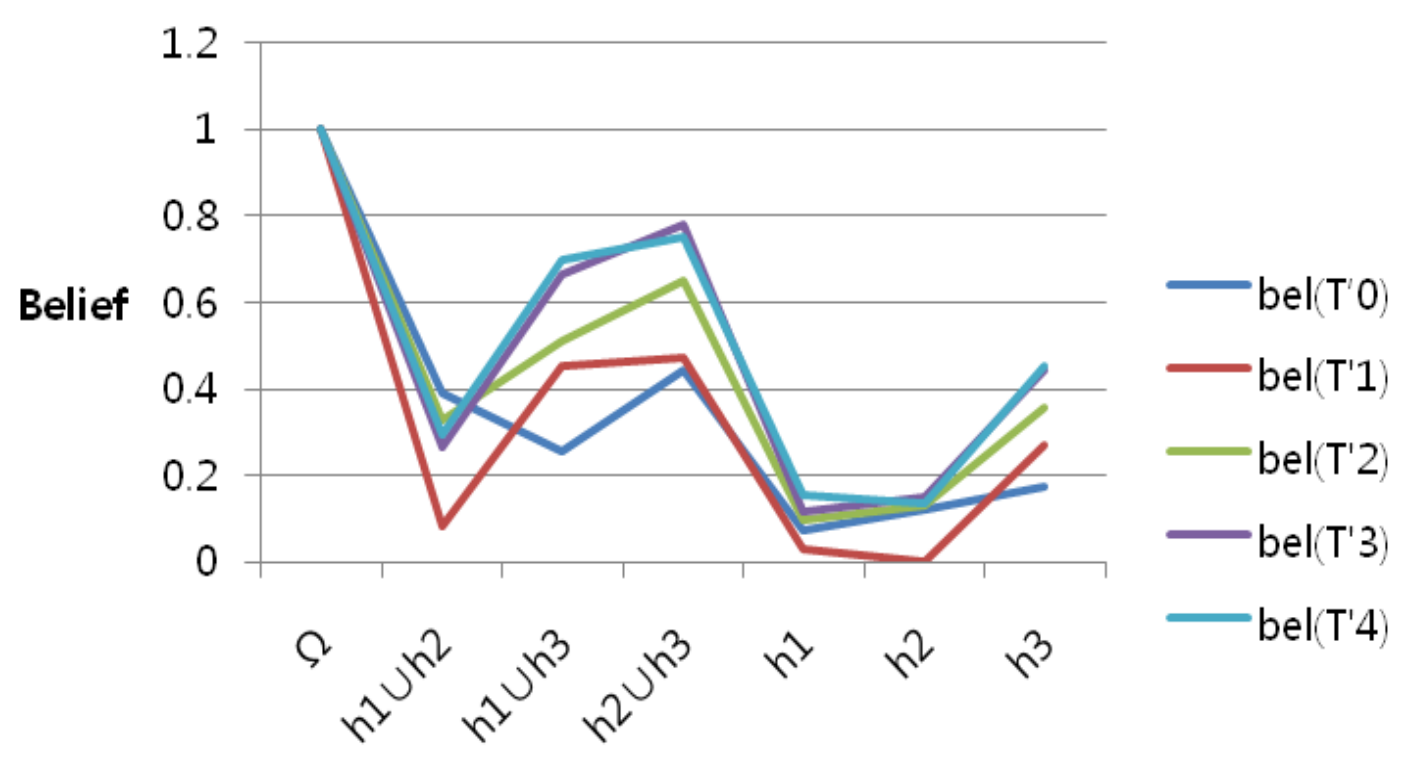

Focal element

Figure 3. A Result of Evaluating about Focal Elements

\section{Conclusion}

In this study, in other to increase the accuracy of the context awareness, temporary storage and utilization for the sensing data were proposed. The criteria to judge whether to use or not the sensing data reported from the sensor in the next time zones was 
presented by saving it temporarily, the data proved to be used in the next time zones should be recycled. At this time, details about which form the data detected and reported in the previous time zones is saved was prepared, the BPA of the focal element should be saved. On using by calling the BPA saved, weights corresponding to the increment of the previous and present time zones should be added. Since then, the belief and uncertainty were calculated by using each focal element's BPA and through this, advanced information can be deduced rather than obtaining context information.

\section{References}

[1] K. Dey, G. D. Abowd and D. Salber, "A Conceptual Framework and a Toolkit for Supporting the Rapid Prototyping of Context-Aware Applications”, Human-Computer Interaction, vol. 16, (2001), pp. 97-166.

[2] D. Salber, A. K. Dey and G. D. Abowd, "The Context Toolkit:Aiding the development of Context-Embeded Applications”, Proceedings of CHI’99, (1999), pp. 434-441

[3] D. H. Suh, J. H. Song and K. S. Oh, "Context Inference for Predicting Cause of Protection Wall Deformation”, International Journal of Smart Home, vol. 6, no. 2, (2012) April.

[4] H.-K. Kim, "Diversity of Mobile Distribution Systems", International Journal of Smart Home, vol. 7, no. 3, (2013), pp. 355-364.

[5] J.-Y. Kim, "Development of Agricultural Cooperatives for Revitalization of the Rural Community - Focused on the Case Study of 'Sunkist'”, International Journal of Smart Home, vol. 7, no. 3, (2013), pp. 293-300.

[6] S.-R. Lee, "A Model to Measure the Success of a Computer-Aided Information System for Prevention of Breast Cancer”, International Journal of Smart Home, vol. 7, no. 2, (2013), pp. 97-104.

[7] E. Hong, "Software Fault-proneness Prediction using Random Forest", International Journal of Smart Home, vol. 6, no. 4, (2012), pp. 147-152.

[8] T. Strang and C Linnhoff-Popien, “A Context Modeling Survey”, Workshop on Advanced Context Modeling, Reasoning and Management, UbiComp, (2004).

[9] S.-W. Ryu, H.-S. Jang, D.-C. Shin and S.-K. Park, “Technical Note of Context-Awareness”, Technical Note of National IT Industry Promotion Agency, Korea, no. 1435, (2010) March 3.

[10] “OWL:Hoolet”, http://owl.man.ac.uk/hoolet/.

[11] "F-OWL", http://sourceforge.net/projects/fowl/.

[12] W. Kevin, C. Sayers and H. Kuno, "Efficient RDF Storages and Retrieval in Jena2”, Proceedings of First International Workshop on Semantic Web and Databases, (2003), pp. 131-151.

[13] Gaia project, http://gaia,cs.uiuc.edu/.

[14] T. Gu, “An Ontology-based Context Model in Intelligent Environments”, Communication Networks and Distributed Systems Modeling and Simulation Conference, San Diego, California, USA, (2004).

[15] T. Hofer, W. Schwinger, M. Picher, G. Leonhartsberger and J. Altman, "Context-Awareness on Mobile Devices the Hydrogen Approach”, $36^{\text {th }}$ Annual Hawaii International Conference on System Sciences, (2003), Hawaii, USA.

[16] U. Rakowsky, "Fundamentals of Dempster-Shafer theory and its applications to system safety and reliability modeling”, International journal of reliability, quality, and safety engineering, vol. 14, no. 6, (2007), pp. 579602.

[17] D. H. Suh and C. K. Ryu, "Multi-sensor Data Fusion Using Weighting Method based on Event Frequency", The Journal of The Korea Institute of Electronic Communication Sciences 2011, vol. 6, no. 4, (2011).

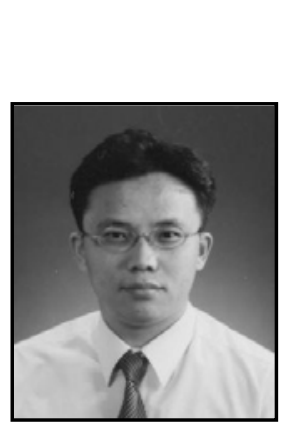

\section{Author}

Younghwan Oh received the M.S. degrees in computer science from Inha University in 1997 and the Ph.D. in computer science from Inha University in 2001. His research interests included in stream data processing and data fusion in wireless sensor network. He is a professor in Korea Nazarene University from 2002. 